\title{
1 Mutually Antagonistic Protein Pairs of Cancer
}

2 Ertuğrul Dalgıç

3 Department of Medical Biology, Zonguldak Bulent Ecevit University School of Medicine,

4 Zonguldak, Turkey

5 Address correspondence to:

6 Ertuğrul Dalgıç

$7 \quad$ Zonguldak Bulent Ecevit Universitesi Tip Fakultesi Dekanligi

8 Tibbi Biyoloji Anabilim Dali

9 Kozlu, Zonguldak, 67600, Turkey

10 Phone: $+90-3722613170$

11 Fax: +90-3722610264

12 E-mail: ertugruld@beun.edu.tr

ORCID: 0000-0003-0536-4447

\section{Abstract}

16 Switch-like behavior of tumorigenesis could be governed by antagonistic gene and protein pairs

17 with mutual inhibition. Unlike extensive analysis of gene expression, search for protein level

18 antagonistic pairs has been limited. Here, potential cancer type specific antagonist protein pairs 
with mutual inhibition were obtained from large scale datasets. Cancer samples or cancer types were compared to retrieve potential protein pairs with contrasting differential expression

21 patterns. Analysis of two different protein expression datasets showed that a few proteins

22 participate in most of the mutually antagonistic relationships. Some proteins with highly

23 antagonistic profile were identified, which could not be attained from a differential expression or

24 a correlation based analysis. The antagonistic protein pairs are sparsely connected by molecular

25 interactions. Glioma, melanoma, and cervical cancer, are more frequently associated with

26 antagonistic proteins than most of the other cancer types. Integrative analysis of mutually

27 antagonist protein pairs contributes to our understanding of systems level changes of cancer.

Introduction

29 Cancer could be viewed as the result of switch like behavior of cells (Mills et al. 2010, Balentine et al. 2011, Torres et al. 2018). Robustness is preserved, as cells transform from normal state to cancer state. The switch like behavior could be best understood by a systems level view of molecular networks. Some specialized molecular interaction patterns, or circuits, could play significant roles for the switch (Siegal-Gaskins et al. 2011). Previously gene circuits which play

34 a role in the bistability of normal network transition to cancer network, were investigated

35 (Shiraishi et al. 2010). Interaction network neighbor genes that show contrasting expression 36 levels for cancer were investigated and shown as candidate players for circuits underlying the 37 switch like behavior.

38 Antagonist protein pairs with mutual inhibition have critical roles for generating bistability 39 (Gardner et al. 2000, Yao et al. 2008, Parashar et al. 2009, Shin et al. 2017, Matsuoka et al. 2018, 
41 each other directly or indirectly. Mutually antagonist proteins show contrasting expression and

42 activity levels in two different stable states. Inhibitory effects of Retinoblastoma (RB) and E2F

43 Transcription Factor on each other, is important for the bistability during a critical cell cycle

44 decision (Yao et al. 2008). A mutual inhibitory mechanism was shown for Phosphatidylinositol

45 3,4,5-trisphosphate (PIP3) and PIP3 phosphatase, providing polarity such that one part of a cell

46 would have PIP3, and another part would have PIP3 phosphatase (Matsuoka et al. 2018). The

47 interaction between PIP3 and its phosphatase provide the bistable behavior of directional

48 movement. Another mutual inhibitory mechanism was shown for RhoA and Rac1, providing

49 bistability for cell migration (Nguyen et al. 2018). Under cellular stress conditions, XIAP-

50 associated factor 1 (XAF1) and metallothionein 2A (MT2A) induce the degradation of each

51 other and this mutual regulation is important for the switch like behavior for tumorigenesis (Shin

52 et al. 2017). Such antagonistic mechanisms were confirmed in expression levels as XAF1 and

53 MT2A were negatively coexpressed in several primary tumors and cell lines (Shin et al. 2017).

54 For the tumors where XAF1 was low and MT2A was high, induction of XAF1 lowered MT2A

55 levels and caused a regression in tumorigenesis. Cyclin dependent kinase 1 (CDK1) and protein

56 phosphatase 2A (PP2A) indirectly inhibit each other and this mutual inhibitory mechanism is

57 critical for mitosis entry switch (Rata et al. 2018). When the mutual inhibitory effects of CDK1

58 and PP2A were disrupted, the switch like entry into mitosis was disrupted. Such examples

59 suggest mutually antagonistic relationships between proteins as important mechanisms for

60 several cellular events including tumorigenesis.

61 Previous studies for the estimation of antagonistic pairs focused on gene expression datasets

62 rather than protein datasets (Shiraishi et al. 2010). However, there is no strong correlation

63 between mRNA and protein expression levels for the majority of human genes (Kosti et al. 
64 2016). Protein level changes, which are not reflected in genomic level could play role in cancer

65 mechanisms (Jarnuczak et al. 2021). Therefore, protein level analysis is required for

66 understanding cancer related antagonistic relationships.

67 Cancer is a complex heterogenic disease as each cancer sample has its own unique gene and

68 protein expression patterns (Axelsen et al. 2007, Kosti et al. 2016, Schneider et al. 2017). There

69 are different tissue specific oncogenic mechanisms (Schneider et al. 2017). Proteins could depict

70 contrasting expression and activity levels in different cancer samples and cancer types.

71 Therefore, two proteins underlying a bistable switch could show opposite behavior in two

72 different cancer samples or cancer types. Here this is exploited to analyze antagonistic pairs

73 across various cancer samples (CS) and cancer types (CT). Protein pairs with antagonistic

74 behavior are such that when the expression level of a protein is up, the expression of the other

75 protein is down and vice versa. Here mutually antagonistic protein pairs (MAPP) are identified

76 by selecting pairs of proteins which are ON (upregulated) and OFF (downregulated) in at least

77 one $\mathrm{CS}$ or $\mathrm{CT}$, and $\mathrm{OFF}$ and $\mathrm{ON}$ in at least one other CS or CT. Some proteins were found to

78 have high number of antagonistic links with other proteins. A few protein pairs were linked by

79 molecular interactions. Members of MAPP could potentially be key players for the switch like

80 behavior of cancer.

\section{Results}

\section{Uncovering MAPP}

83 MAPP were explored by selecting protein pairs with opposing differential expression profiles;

84 which are $\mathrm{ON}$ and $\mathrm{OFF}$ respectively in at least one CS or CT, and OFF and ON in at least one 85 other CS or CT (Figure 1A-B). 342 distinct MAPP among 80 proteins were identified in The 
Cancer Proteome Atlas (TCPA) dataset based on comparison of the CS (Supplementary Table 1).

87 On the other hand, 341 distinct protein pairs were found by comparing the CT of the TCPA dataset (Supplementary Table 1). All of the CT based MAPP were the same with the CS based pairs except for the missing HSP70-PKCPANBETAII_pS660 pair. Significance of the MAPP was analyzed by randomization tests. $187 \mathrm{CS}$ based pairs and $111 \mathrm{CT}$ based pairs are significant (FDR corrected p-value < 0.05) (Supplementary Table 1). 97 pairs are significant in both CS and CT based MAPP. Network view of the combined significant MAPP of CS and CT based analyses shows that a few proteins such as EPPK1, MYH11, ESR1, CAV1, ANXA1, PEA15, TFRC, TUBA1B, GAPDH, and SRC are highly connected whereas most of the proteins are lowly connected in the combined list of MAPP which include both CS and CT based pairs (Figure 2A, Supplementary Figure 1). Degree distribution of the MAPP networks are similar for individual CS or CT based analyses (Supplementary Figure 2).

Human Protein Atlas (HPA) dataset provides only cancer type based values unlike TCPA. However, it has a higher coverage of human proteome. There are 19651 unique proteins in the HPA dataset and 217 unique proteins in the TCPA dataset based on NCBI gene symbols. All of the 217 TCPA proteins are also present in the HPA dataset. MAPP were defined based on the comparison of the HPA cancer types. 148 distinct MAPP among 90 proteins were identified (Supplementary Table 2). 142 pairs are significant based on the randomization test (FDR corrected p-value < 0.05). Remarkably, there is no common protein pair between the TCPA and the HPA MAPP lists, and GAPDH is the only common protein. HPA MAPP network has a few highly connected proteins such as LRRC26, ABHD3, SPIDR, PKP3, PC, and KRT5 while most

107 of the proteins are lowly connected (Figure 2B, Supplementary Figure 3). Thus, analyses of both datasets showed that a few proteins participate in most of the mutually antagonistic relationships. 


\section{Comparison of Antagonistic Relationships to Differential Expression and Correlation}

Next, assessment of cancer proteins for mutual antagonism was compared to the typical analyses of differential expression and coexpression. Parameters for MAPP, differential expression and expression correlation (PCPP; positive correlation based protein pairs, NCPP; negative correlation based protein pairs) were defined for each protein in the TCPA and HPA datasets (see Methods). MAPP, PCPP, and NCPP based differential ratio values were calculated as the difference of their ratio values from the differential expression based ratio value for each protein. The profile of mutual antagonism based values differs from positive and negative correlation for the CS and CT based analyses of the TCPA and HPA datasets (Figure 3, Supplementary Tables 3-5). For the CT based analyses of both datasets, MAPP based values of some proteins are considerably different from differential expression unlike correlation based values. Unlike PCPP and NCPP, MAPP based differential ratio values are highly positive for some proteins. Therefore, the current analysis provides molecules with distinct antagonistic characteristics which could not be obtained from a differential expression or a correlation based analysis.

TCPA proteins such as EPPK1, MYH11, TUBA1B, ANXA1, TFRC, PEA15, ESR1, PEA15, CAV1, GAPDH, and CCNB1 have highly positive MAPP based differential ratio values in the CS or CT based analyses, thus are highly antagonistic with other proteins (Table 1). Some proteins such as TUBA1B and ANXA1 are common to the CS and CT based lists, others such as EPPK1 and MYH11 are specific to only one of the lists. Although EPPK1 and MYH11 have the highest differential expression values in the CS based analysis, they have positive MAPP based differential ratio values only in the CT based analysis (Supplementary Tables 3-4). On the other hand, GSK3ALPHABETA_pS21S9 (GSK3A, GSK3B) and ATM have relatively lower differential expression values but positive MAPP based ratio values. Although, a similar profile 
132

was observed for both CS and CT based analyses of differential ratio values, some proteins have disparate values. Also most of the top proteins with the highest positive and negative correlation numbers do not have highly positive MAPP based differential ratio values (Supplementary tables 3-4).

HPA proteins such as LRRC26, PC, ABHD3, PKP3, SPIDR, KRT5, KRT17, TRPS1, RNASEH2C, and GIGYF1 have highly positive MAPP based differential ratio values (Table 2,

Supplementary Table 5). None of the top proteins with the highest differential expression number, positive and negative correlation numbers have positive MAPP based differential ratio values. Analyses of both TCPA and HPA datasets showed that mutual antagonism analysis provides unique proteins. Differential expression or correlation based values do not estimate the mutual antagonistic parameters of a protein.

\section{Interaction Networks of MAPP}

Protein-protein interactions (PPI) and protein-DNA interactions (PDI) between the antagonist proteins were investigated. Significant MAPP of the TCPA and HPA datasets were integrated with human PPI and PDI datasets (see Methods). Only 4 pairs from both CS and CT based MAPP of the TCPA dataset have direct protein-protein interactions; STAT5A-ESR1, CAV1SRC, CTNNB1-YAP1, and MAP2K1-YAP1. CS based MAPP also has CAV1-SQSTM1 as a directly interacting pair which is not a significant CT based MAPP. Only one pair from both CS and CT based MAPP has a direct PDI; STAT5A-ESR1, where STAT5A is the TF of ESR1 gene. 2 other PDI are present in the CS based MAPP; AR-ANXA1 and ESR1-CTNNB1; where AR and ESR1 are the TF of ANXA1 and CTNNB1 genes respectively. STAT5A-ESR1 is the only pair which has both direct PPI and PDI. Furthermore, more TCPA MAPP have indirect PPI 
154 between them (Figure 4, Supplementary Table 6). Among the 210 significant TCPA MAPP

155 (based on NCBI symbols), 34 CS and CT based pairs, 35 only CS based pairs, and 5 only CT

156 based pairs have indirect interactions. ESR1, CAV1, SRC, YAP1, CTNNB1, GAPDH, and

157 CCNB1 are highly connected in the PPI integrated network (Figure 4). On the other hand, none

158 of the significant HPA MAPP have direct PPI or PDI between them. Furthermore, only one pair,

159 which is HSPB1-PARD6A, has indirect PPI (Supplementary Table 7). Overall, there are very

160 few antagonist pairs with direct molecular interactions. Some TCPA pairs have indirect

161 interactions and HPA pairs have no direct or indirect interactions between them except for one.

\section{Cancer Pairs of MAPP}

163 MAPP definition of this study was based on the comparison of expression levels of two proteins

164 in two different CS or CT (Figure 1). Finally CT pairs, which were used for the designation of

165 significant MAPP of the TCPA and HPA datasets, were listed (Supplementary Tables 8-9). CT

166 of significant MAPP of the TCPA and HPA datasets, include some cancer more frequently than

167 most of the other cancers (Figure 5). LGG (Brain Lower Grade Glioma), SARC (Sarcoma),

168 CESC (Cervical squamous cell carcinoma and endocervical adenocarcinoma), SKCM (Skin

169 Cutaneous Melanoma) and BLCA (Bladder Urothelial Carcinoma) have higher counts of

170 appearance in the CT lists of the TCPA dataset. Breast cancer, testis cancer, melanoma, glioma,

171 cervical cancer, and endometrial cancer have high counts in the CT lists of the HPA dataset.

172 None of these cancers were overrepresented in the datasets (Supplementary Table 10-11).

173 Glioma (LGG), melanoma (SKCM), and cervical cancer (CESC) are frequently used by the 174 antagonist proteins of both datasets. 


\section{Discussion}

177

178

179

180

181

182

183

184

185

186

187

188

189

190

191

192

193

194

195

196

197

In this study, samples and types of cancer are compared among themselves to identify pairs of proteins with contrasting levels, unlike typical studies focusing on only normal and cancer comparison. For some proteins, MAPP associated ratio values, their associated MAPP count relative to all other proteins, appear to be distinctive. Such information for those proteins cannot be received from differential equation or correlation based analyses. Even, negative correlation values of such proteins are not distinguishing. Therefore, examination of mutual antagonism by comparing CS or CT to each other, provides unique proteins with distinct characteristics. MAPP analyses showed that a few proteins participate in most of the mutually antagonistic relationships, and these proteins have generally positive MAPP based ratio values. EPPK1, MYH11, TUBA1B, ANXA1, TFRC, PEA15, ESR1, PEA15, CAV1, GAPDH, CCNB1, LRRC26, ABHD3, SPIDR, PKP3, PC, and KRT5 are some of the proteins with a high number appearances in MAPP networks and distinctive MAPP based ratio values.

STAT5A and ESR1 (ER alpha) pair is in both CS and CT based MAPP of the TCPA dataset analysis and has both PPI and PDI. There is experimental evidence for potential antagonistic or redundant relationships between them (Wang and Cheng 2004, Miermont et al. 2010). Another MAPP of the TCPA is SRC-CAV1. They also have a direct interaction. The relationship between SRC and CAV1 was shown previously (Thomas et al. 2011). EPPK1-PEA15_pS116 pair has the highest number of occurrences in the TCPA dataset analysis. Therefore a potential antagonistic relationship between the two proteins could be investigated. Currently there is no experimental evidence for a direct relationship between these two proteins. PEA15_pS116 specifically represents the form of PEA15 (proliferation and apoptosis adaptor protein 15) which is phosphorylated at Ser-116, thus actively inhibiting apoptosis (Renganathan et al. 2005). EPPK1 
(Epiplakin 1), knock-down is associated with cell migration and proliferation (Kokado et al. 2016). Therefore, EPPK1 and PEA15 might have mutually exclusive anti-proliferative effects in several cancer samples. Since the current study is based on the comparison of CS or CT to each other, identified pairs could have roles in bistability or they could be dispensable for each other. Thus, determination of their mechanistic roles for cancer needs further evidence. a high number of proteins, however, only the values for distinct cancer types could be analyzed. across various cancer types. There is no common pair between the significant MAPP of TCPA and HPA. This could be due to different sizes of the datasets. Expression scores of the two network of HPA also does not have any direct PPI and has only one indirect PPI, which could be

211 due to incomplete interaction databases. Besides, some pairs might be connected by more 212 indirect connections.

213 HPA dataset values ignore modifications such as phosphorylation which could determine the 214 activity of some proteins. On the other hand, TCPA dataset values have some modification215 specific values. Therefore, the reason for the absence of some TCPA MAPP from HPA MAPP 216 could be due to the negligence of such modification-specific values. When several normal and 217 cancer tissues were examined, 64-80\% of cases with significant expression differences, had low 218 XAF1 and high MT2A levels confirming the role of antagonistic mechanism between these 219 proteins in cancer (Shin et al. 2017). Therefore when using generic datasets like HPA, some 220 antagonistic pairs will be missed. In order to capture a larger scale identification of such pairs, individual sample based datasets are needed. Furthermore, because of the heterogenic nature of 
222 tumor tissues, single cell based proteomics datasets might be needed for elucidating all

223 antagonistic pairs. Noting the importance of chemical modifications for protein function,

224 phosphorylation specific measurements provide a more biologically meaningful perspective. The

225 pairs obtained by the analysis of HPA dataset ignores such modifications, therefore the high

226 expression of a certain protein does not guarantee an increase in its activity. Therefore, such

227 pairs should be further examines computationally and experimentally for changes in their

228 activity, for if there is actually an antagonistic relationship between the two proteins with respect

229 to their activities. With the availability of datasets of bigger size, in terms of the number of

230 proteins and the number of samples, in the future, could enable us to find a higher number of

231 significant protein pairs that could play a role in cancer.

232 Different bistability generating feedback circuits could work together to have a robust control on

233 cellular events (Rata et al. 2018). Similarly different antagonistic pairs could also operate

234 cooperatively to achieve important transitions such as tumorigenesis. The potential bistability

235 generating antagonistic circuits identified here could work in different combinations for different

236 cases. Frequently observed proteins could have important roles for different cancer types.

237 Elucidating such mechanisms could be critical for systems level understanding of cancer.

\section{Methods}

\section{Prediction of MAPP across Cancer Samples}

240 Cancer sample expression values for different proteins were obtained from The Cancer Proteome

241 Atlas (TCPA) database version 4.2 (https://tcpaportal.org) (Li et al. 2013). TCPA dataset values

242 are based on Reverse phase protein arrays (RPPA). TCPA has 4 levels of data processing steps.

243 Level 4 values have normalized values such that different batch effects were removed and values 
244 for different cancer samples were merged. Here, relative quantitative values of Pan-Cancer Level

2454 TCPA dataset were used to assess differential expression of 217 proteins (including different 246 modifications of the same protein, for instance P27_pT198, a phosphorylated form of P27, was

247 treated as a separate protein, see Supplementary Information). The dataset has a total of 7694

248 cancer samples for 32 different cancer types. The threshold values -2 and 2 were chosen to 249 define differentially expressed proteins in the TCPA dataset (see Supplementary Information).

250 Proteins with an expression value higher than 2 were defined as $\mathrm{ON}$ and the ones with an

251 expression value lower than -2 were defined as OFF for each cancer sample. Then, mutually 252 antagonistic protein pairs (MAPP) were defined as those proteins which are ON-OFF in at least 253 one sample, and OFF-ON in at least one other sample ignoring the cancer type of the sample.

254 Next, a permutation test was done by shuffling the expression values of each sample. Antagonist 255 pairs were similarly found in the random datasets. The number of sample pairs, for which the 256 antagonist relationship was defined for each protein pair, was compared to random values and p257 values were defined as the fraction of random iterations with an equal or higher number of 258 samples pairs. Multiple testing correction of the p-values was done by False Discovery Rate 259 (FDR). 0.05 was chosen as the FDR correct p-value cutoff to define the significant MAPP.

\section{Prediction of MAPP across Cancer Types}

261 TCPA dataset also has cancer type information for all samples. Therefore, a similar analysis was

262 also done based on cancer types, in which differential expression of a protein in at least one of 263 the samples belonging to a cancer type was considered when searching for protein pairs with 264 contrasting ON-OFF trends. MAPP were defined as those proteins which are ON-OFF in at least 
cancer type, and OFF-ON in at least one other cancer type. Permutation test based p-values for protein pairs were defined similarly and multiple testing correction was done by FDR. tissue and cancer expression (pathology) values for different proteins were obtained from HPA database version 20. Normal tissue and pathology datasets were used to determine semi-

271 quantitative differential expression of proteins. Manual scoring of immunohistochemistry 272 analysis is shown as 'not detected', 'low', 'medium', and 'high'. Cancer dataset has scoring 273 values for 15308 proteins and 17 different cancer types after removing rows with missing values and very low number of measurements (see Supplementary Information). There are multiple measurements for different cases of a cancer type and there is a variation among the different

276 samples. However, there is a single scoring value for normal tissue cell type samples. To 277 determine the differential expression of a protein, proteins with binary expression (present or 278 absent) in cancer and normal were determined. For the pathology dataset values, the Cancer 279 Expression parameter (CE) was defined.

$$
C E=\frac{(\mathrm{w} \times \mathrm{h}+\mathrm{m})-(\mathrm{l}+\mathrm{w} \times \mathrm{n})}{\mathrm{w} \times \mathrm{h}+\mathrm{m}+\mathrm{l}+\mathrm{w} \times \mathrm{n}} \#(1)
$$

In equation 1; $\mathrm{n}, \mathrm{l}, \mathrm{m}$, and $\mathrm{h}$ are the number of samples with 'not detected', 'low', 'medium',

281 'high' expression respectively, and w is the weight for 'not detected' and 'high' scores. As CE 282 gets bigger or lower than 0, protein expression gets more definitive. Absolute CE threshold value 283 was set to 0.5 and w was set to 2 (see Supplementary Information). Proteins with the CE value 284 lower than -0.5 was defined as absent and the ones with the CE value higher than 0.5 was 285 defined as present. Proteins with binary (present/absent) expression in all cancer types were 
specified by using the CE values. Secondly, differentially expressed proteins were determined by comparing the cancer expression to normal. The normal dataset has scoring values for 1210013500 proteins, for different cell types from different tissues. Unlike the cancer dataset, the normal dataset has only a single value, therefore, proteins with 'not detected' score was defined as absent, and the ones with 'high' score was defined as present. Cancer types and tissues and cell types were matched manually (see Supplementary Information). For each cancer typenormal tissue match, proteins which are present in cancer and absent in normal tissue were defined as $\mathrm{ON}$ (upregulated), and proteins which are absent in cancer and present in normal tissue was defined as OFF (downregulated). MAPP were defined as those proteins which are $\mathrm{ON}-\mathrm{OFF}$ in at least one cancer type, and OFF-ON in at least one other cancer type. were assigned for each protein as the distribution of the total values for each protein $(h+m+l+n)$

299 iterations, for which the number antagonist relationships across all cancer type pairs was not (FDR). 0.05 was chosen as the FDR correct p-value cutoff to define the significant MAPP.

\section{Differential Ratio Values of Protein Pairs and Cancer Types}

For the proteins which were defined as differentially expressed in TCPA or HPA datasets, weighted ratio values based on differential expression, positive or negative correlation and protein was defined as the number of CS (or CT) for which differential expression was observed. 
Positive correlation based protein pairs (PCPP) and negative correlation based protein pairs (NCPP) were analyzed. For correlation analysis, Jaccard index based correlation value for each protein was calculated as below.

$$
\begin{gathered}
P C_{i}=\sum_{k \in A} \frac{\left(u_{i} \cap u_{k}\right)+\left(d_{i} \cap d_{k}\right)}{\left(u_{i} \cup u_{k}\right)+\left(d_{i} \cup d_{k}\right)} \#(2) \\
N C_{i}=\sum_{k \in A} \frac{\left(u_{i} \cap d_{k}\right)+\left(d_{i} \cap u_{k}\right)}{\left(u_{i} \cup d_{k}\right)+\left(d_{i} \cup u_{k}\right)} \#(3)
\end{gathered}
$$

where $\mathrm{PC}_{\mathrm{i}}$ is the positive correlation value, and $\mathrm{NC}_{\mathrm{i}}$ is the negative correlation value of protein $\mathrm{i}$, of CS (or CT) in which protein $\mathrm{i}$ and protein $\mathrm{k}$ are $\mathrm{OFF}, \mathrm{A}$ is the set of all proteins except for the protein i.

Finally, the mutually antagonistic value (MAPP number) for each protein was calculated the sum

315 of all pairs in which the protein was defined as being in a mutually antagonistic pair. Ratio

316 values for differential expression, correlation and mutual antagonism of each protein were

317 calculated by dividing the individual value of each protein to the sum for all proteins.

318 Differential ratio values for PCPP, NCPP and MAPP were calculated as the difference of the

319 correlation and the mutual antagonism based values from the differential expression based 320 values. Both CS and CT based values were calculated for TCPA and only CT based values were 321 calculated for HPA. 
323 Molecular interaction neighborhood of significant MAPP was analyzed for both TCPA and HPA

324 datasets. BioGrid Physical Interaction Dataset Version 4.3.194 was used for human protein-

325 protein interactions (Stark et al. 2006, Oughtred et al. 2021). TRRUST (release note 2018.04.16)

326 and The Human Transcriptional Regulation Interactions database (HTRIdb) were used for

327 human transcriptional (PDI) interactions (Han et al. 2015, Han et al. 2018, Bovolenta et al.

328 2012). Antibody based protein names of TCPA were converted to NCBI Gene Symbols, which

329 could be integrated with protein-protein and transcriptional interactions (Supplementary Table

330 12). Direct (first degree) and indirect (second degree) protein-protein interactions of MAPP were

331 explored. One TCPA name could match to a maximum of 3 different NCBI Gene Symbols,

332 therefore all possible interactions of the matching Symbols were considered separately. For

333 transcriptional interactions, TRRUST and HTRIdb database pairs were combined in order to

334 have a more comprehensive dataset. Then, directional transcription factor-gene interactions of

335 MAPP were explored.

\section{Software and Code Availability}

$337 \mathrm{R}$ scripts are available at https://github.com/ertuda/MAPP. Network visualization was done by 338 Cytoscape (Shannon et al. 2003).

\section{References}

340 Axelsen JB, Lotem J, Sachs L, Domany E. Genes overexpressed in different human solid cancers 341 exhibit different tissue-specific expression profiles. Proc Natl Acad Sci U S A. 2007 Aug 342 7;104(32):13122-7. doi: 10.1073/pnas.0705824104. 
343 Balentine CJ, Berger DH, Liu SH, Chen C, Nemunaitis J, Brunicardi FC. Defining the cancer

344 master switch. World J Surg. 2011 Aug;35(8):1738-45. doi: 10.1007/s00268-010-0941-2. PMID:

34521286716.

346 Bovolenta LA, Acencio ML, Lemke N. HTRIdb: an open-access database for experimentally

347 verified human transcriptional regulation interactions. BMC Genomics. 2012 Aug 17;13:405.

348 doi: 10.1186/1471-2164-13-405.

349 Gardner TS, Cantor CR, Collins JJ. Construction of a genetic toggle switch in Escherichia coli.

350 Nature. 2000 Jan 20;403(6767):339-42. doi: 10.1038/35002131.

351 Han H, Cho JW, Lee S, Yun A, Kim H, Bae D, Yang S, Kim CY, Lee M, Kim E, Lee S, Kang B,

352 Jeong D, Kim Y, Jeon HN, Jung H, Nam S, Chung M, Kim JH, Lee I. TRRUST v2: an expanded

353 reference database of human and mouse transcriptional regulatory interactions. Nucleic Acids

354 Res. 2018 Jan 4;46(D1):D380-D386. doi: 10.1093/nar/gkx1013.

355 Han H, Shim H, Shin D, Shim JE, Ko Y, Shin J, Kim H, Cho A, Kim E, Lee T, Kim H, Kim K,

356 Yang S, Bae D, Yun A, Kim S, Kim CY, Cho HJ, Kang B, Shin S, Lee I. TRRUST: a reference

357 database of human transcriptional regulatory interactions. Sci Rep. 2015 Jun 12;5:11432. doi:

$358 \quad 10.1038 /$ srep11432.

359 Jarnuczak AF, Najgebauer H, Barzine M, Kundu DJ, Ghavidel F, Perez-Riverol Y,

360 Papatheodorou I, Brazma A, Vizcaíno JA. An integrated landscape of protein expression in

361 human cancer. Sci Data. 2021 Apr 23;8(1):115. doi: 10.1038/s41597-021-00890-2. 
Kokado M, Okada Y, Miyamoto T, Yamanaka O, Saika S. Effects of epiplakin-knockdown in cultured corneal epithelial cells. BMC Res Notes. 2016 May 20;9:278. doi: 10.1186/s13104-0162082-7.

Kosti I, Jain N, Aran D, Butte AJ, Sirota M. Cross-tissue Analysis of Gene and Protein Expression in Normal and Cancer Tissues. Sci Rep. 2016 May 4;6:24799. doi: $10.1038 /$ srep24799.

Li J, Lu Y, Akbani R, Ju Z, Roebuck PL, Liu W, Yang JY, Broom BM, Verhaak RG, Kane DW, Wakefield C, Weinstein JN, Mills GB, Liang H. TCPA: a resource for cancer functional proteomics data. Nat Methods. 2013 Nov;10(11):1046-7. doi: 10.1038/nmeth.2650.

Matsuoka S, Ueda M. Mutual inhibition between PTEN and PIP3 generates bistability for polarity in motile cells. Nat Commun. 2018 Oct 26;9(1):4481. doi: 10.1038/s41467-018-068560.

Miermont AM, Parrish AR, Furth PA. Role of ERalpha in the differential response of Stat5a loss in susceptibility to mammary preneoplasia and DMBA-induced carcinogenesis. Carcinogenesis. 2010 Jun;31(6):1124-31. doi: 10.1093/carcin/bgq048.

Mills AA. Throwing the cancer switch: reciprocal roles of polycomb and trithorax proteins. Nat Rev Cancer. 2010 Oct;10(10):669-82. doi: 10.1038/nrc2931.

Nguyen LK, Kholodenko BN, von Kriegsheim A. Rac1 and RhoA: Networks, loops and bistability. Small GTPases. 2018 Jul 4;9(4):316-321. doi: 10.1080/21541248.2016.1224399.

Oughtred R, Rust J, Chang C, Breitkreutz BJ, Stark C, Willems A, Boucher L, Leung G, Kolas N, Zhang F, Dolma S, Coulombe-Huntington J, Chatr-Aryamontri A, Dolinski K, Tyers M. The 
BioGRID database: A comprehensive biomedical resource of curated protein, genetic, and chemical interactions. Protein Sci. 2021 Jan;30(1):187-200. doi: 10.1002/pro.3978.

Rata S, Suarez Peredo Rodriguez MF, Joseph S, Peter N, Echegaray Iturra F, Yang F,

Madzvamuse A, Ruppert JG, Samejima K, Platani M, Alvarez-Fernandez M, Malumbres M,

387 Earnshaw WC, Novak B, Hochegger H. Two Interlinked Bistable Switches Govern Mitotic

388 Control in Mammalian Cells. Curr Biol. 2018 Dec 3;28(23):3824-3832.e6. doi:

389 10.1016/j.cub.2018.09.059.

390 Renganathan H, Vaidyanathan H, Knapinska A, Ramos JW. Phosphorylation of PEA-15

391 switches its binding specificity from ERK/MAPK to FADD. Biochem J. 2005 Sep 15;390(Pt

392 3):729-35. doi: 10.1042/BJ20050378.

Schneider G, Schmidt-Supprian M, Rad R, Saur D. Tissue-specific tumorigenesis: context 394 matters. Nat Rev Cancer. 2017 Apr;17(4):239-253. doi: 10.1038/nrc.2017.

Shannon P, Markiel A, Ozier O, Baliga NS, Wang JT, Ramage D, Amin N, Schwikowski B, Ideker T. Cytoscape: a software environment for integrated models of biomolecular interaction networks. Genome Res. 2003 Nov;13(11):2498-504. doi: 10.1101/gr.1239303. antagonism as a molecular switch in cell-fate decisions under stressful conditions. Proc Natl Acad Sci U S A. 2017 May 30;114(22):5683-5688. doi: 10.1073/pnas.1700861114.

401 Shiraishi T, Matsuyama S, Kitano H. Large-scale analysis of network bistability for human 402 cancers. PLoS Comput Biol. 2010 Jul 8;6(7):e1000851. doi: 10.1371/journal.pcbi.1000851. 
403

404

405

406

407

408

409

410

411

412

413

414

415

416

417

418

419

420

421

422
Siegal-Gaskins D, Mejia-Guerra MK, Smith GD, Grotewold E. Emergence of switch-like behavior in a large family of simple biochemical networks. PLoS Comput Biol. 2011 May;7(5):e1002039. doi: 10.1371/journal.pcbi.1002039.

Stark C, Breitkreutz BJ, Reguly T, Boucher L, Breitkreutz A, Tyers M. BioGRID: a general repository for interaction datasets. Nucleic Acids Res. 2006 Jan 1;34(Database issue):D535-9. doi: $10.1093 /$ nar/gkj109.

Thomas S, Overdevest JB, Nitz MD, Williams PD, Owens CR, Sanchez-Carbayo M, Frierson HF, Schwartz MA, Theodorescu D. Src and caveolin-1 reciprocally regulate metastasis via a common downstream signaling pathway in bladder cancer. Cancer Res. 2011 Feb 1;71(3):83241. doi: 10.1158/0008-5472.CAN-10-0730.

Torres J, Monti R, Moore AL, Seimiya M, Jiang Y, Beerenwinkel N, Beisel C, Beira JV, Paro R. A switch in transcription and cell fate governs the onset of an epigenetically-deregulated tumor in Drosophila. Elife. 2018 Mar 21;7:e32697. doi: 10.7554/eLife.32697.

Uhlén M, Fagerberg L, Hallström BM, Lindskog C, Oksvold P, Mardinoglu A, Sivertsson Å, Kampf C, Sjöstedt E, Asplund A, Olsson I, Edlund K, Lundberg E, Navani S, Szigyarto CA, Odeberg J, Djureinovic D, Takanen JO, Hober S, Alm T, Edqvist PH, Berling H, Tegel H, Mulder J, Rockberg J, Nilsson P, Schwenk JM, Hamsten M, von Feilitzen K, Forsberg M, Persson L, Johansson F, Zwahlen M, von Heijne G, Nielsen J, Pontén F. Proteomics. Tissuebased map of the human proteome. Science. 2015 Jan 23;347(6220):1260419. doi: 10.1126/science.1260419. PMID: 25613900. 
$423 \mathrm{Wu} \mathrm{M}$, Liu L, Chan C. Identification of novel targets for breast cancer by exploring gene

424 switches on a genome scale. BMC Genomics. 2011 Nov 3;12:547. doi: 10.1186/1471-2164-12-

425547.

426 Yao G, Lee TJ, Mori S, Nevins JR, You L. A bistable Rb-E2F switch underlies the restriction

427 point. Nat Cell Biol. 2008 Apr;10(4):476-82. doi: 10.1038/ncb1711.

428 Wang Y, Cheng CH. ERalpha and STAT5a cross-talk: interaction through C-terminal portions of 429 the proteins decreases STAT5a phosphorylation, nuclear translocation and DNA-binding. FEBS

430 Lett. 2004 Aug 13;572(1-3):238-44. doi: 10.1016/j.febslet.2004.06.098. 
A

CS1/CT1

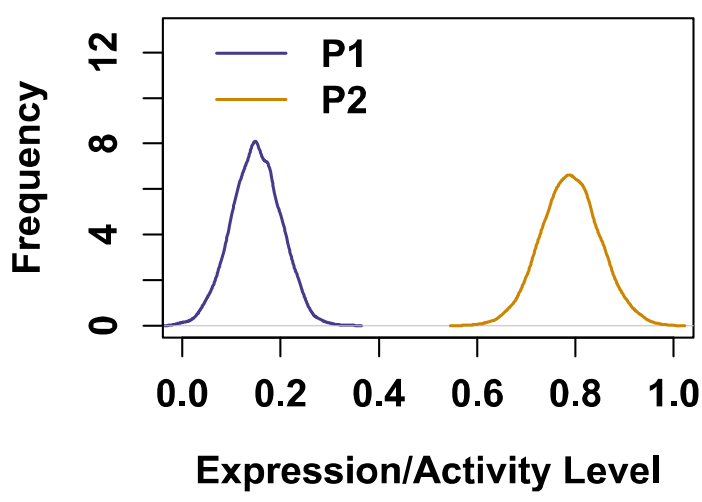

B

CS2/CT2

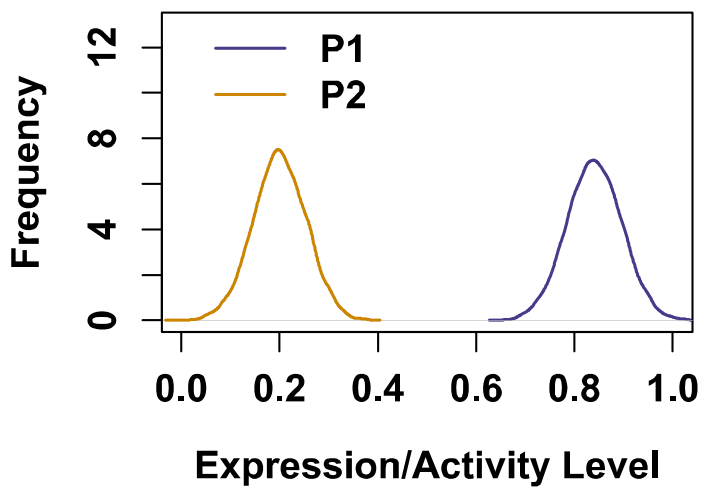

C

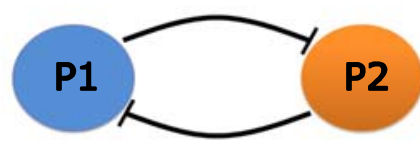

434 Figure 1. Hypothetical MAPP expression and interaction motif (A) Expression or activity

435 levels of two proteins (P1 and P2) which have a mutually antagonistic relationship with each 436 other across two different cancer samples (CS1 and CS2) or cancer types (CT1 and CT2). (B)

437 Potential direct or indirect interactions between the proteins in a MAPP. One explanation for the 

other one in the pair.

\section{A Combined TCPA MAPP Network}

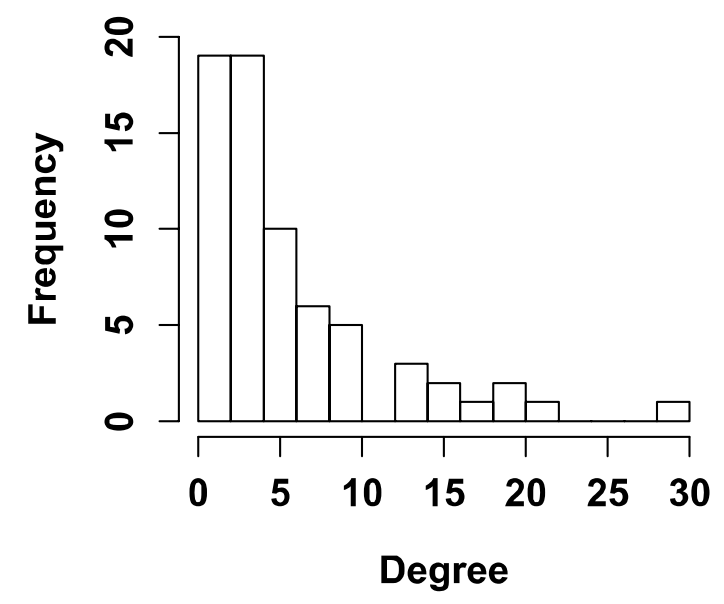

B

HPA MAPP network

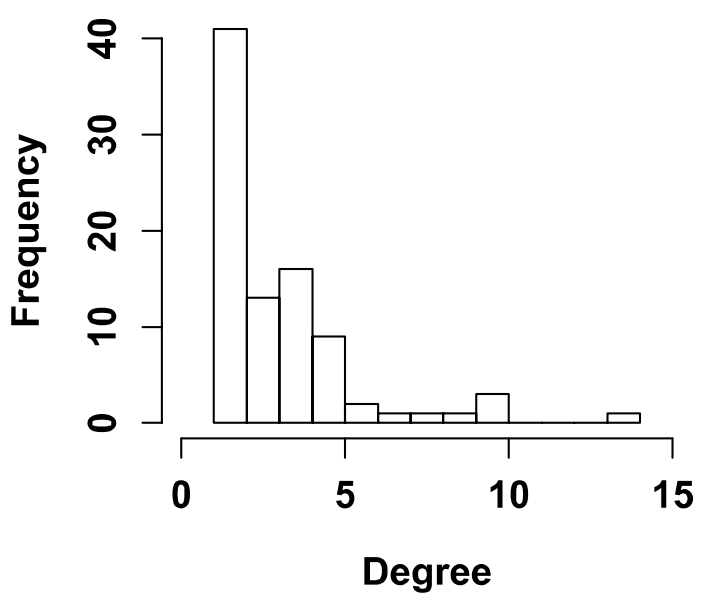

441 Figure 2. Degree distribution of MAPP networks (A) CS and CT based MAPP networks of

442 the TCPA dataset were combined. Degree values (number of links) of the unique MAPP of the

443 combined list were used for the distribution. (B) MAPP network of the HPA dataset were used

444 for the distribution. 


\section{A CS Based Analysis of TCPA}

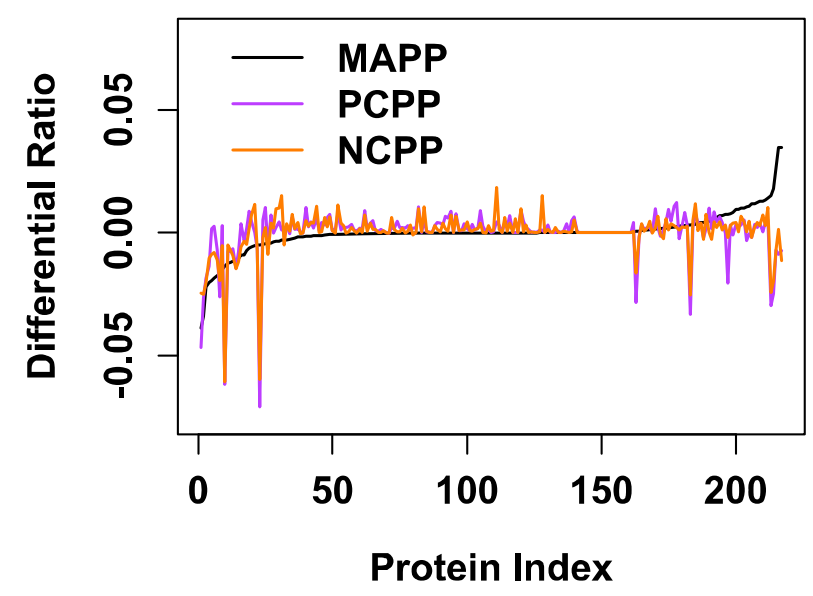

B CT Based Analysis of TCPA

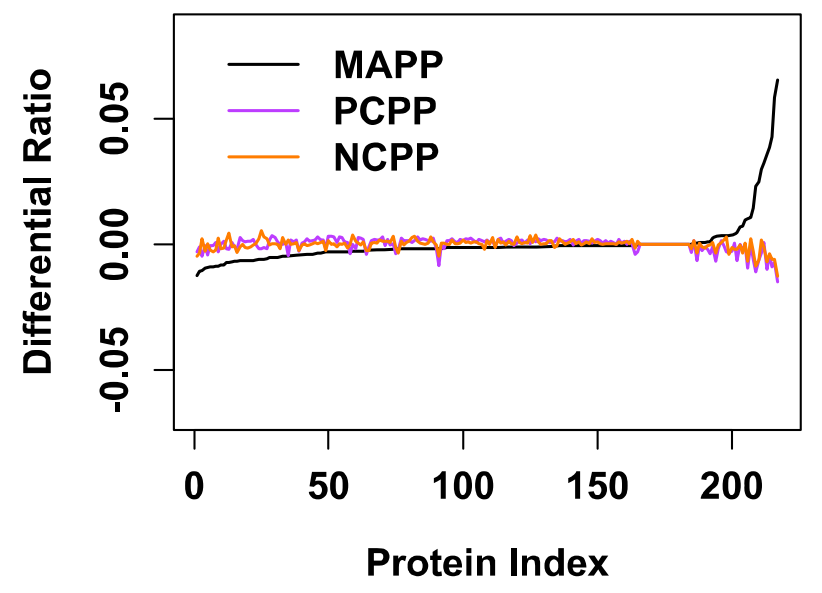

C

CT Based Analysis of HPA

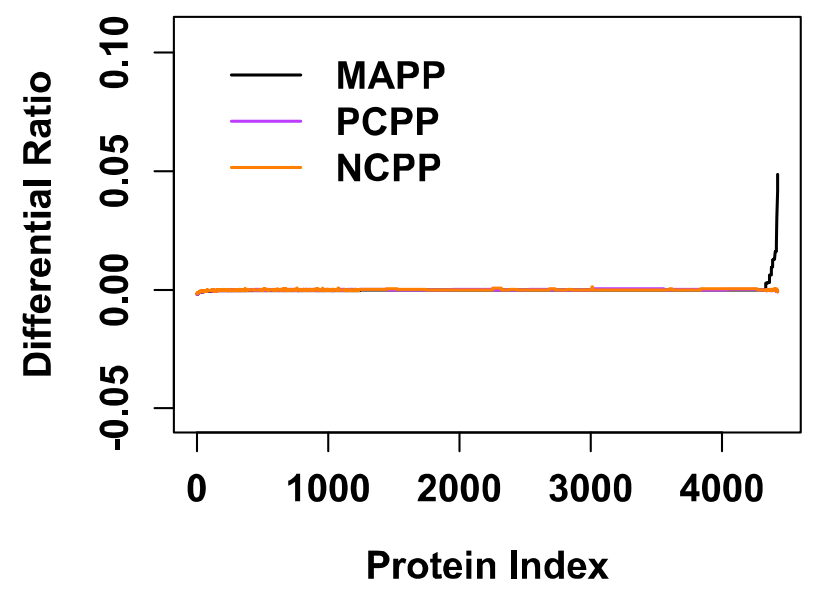



pairs (NCPP) from the weighted ratio value for differential expression. Protein index is sorted

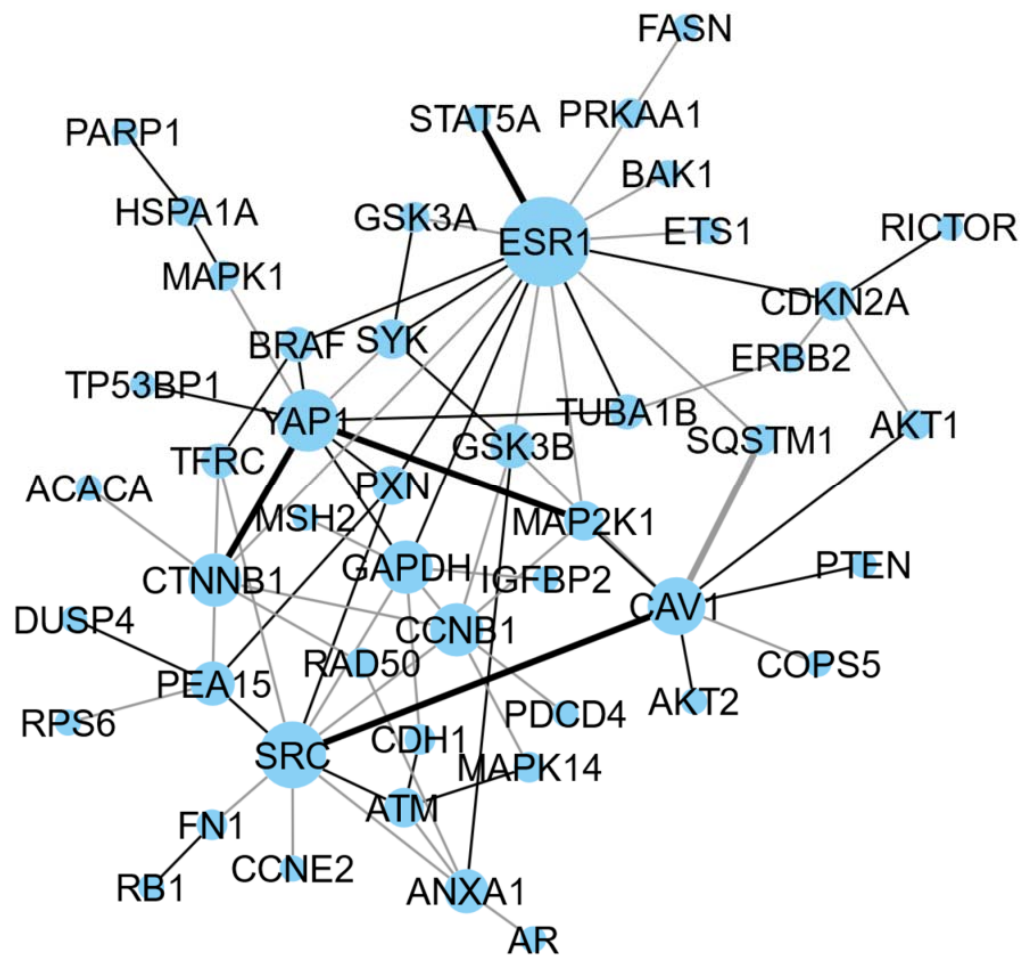




\section{A Cancer Frequencies of TCPA}

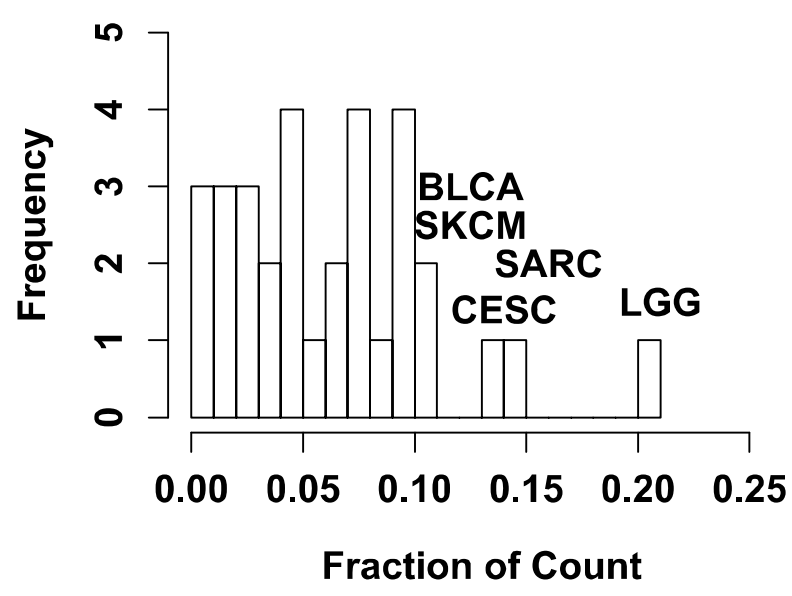

B

\section{Cancer Frequencies of HPA}

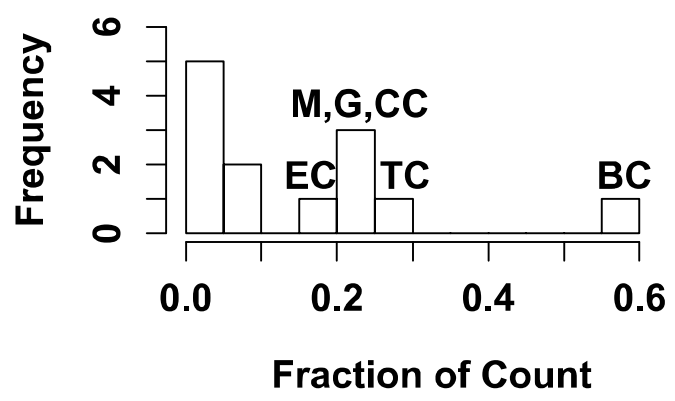

459

460 Figure 5. Frequencies of CT pairs associated with significant MAPP (A) Frequency of CT

461 counts for the TCPA dataset. (B) Frequency of counts for the HPA dataset. High ranking

462 frequent cancers were shown. (BC: breast cancer, TC: testis cancer, CC: cervical cancer, G:

463 glioma, M: melanoms, EC: endometrial cancer) 
Table 1. Top ranked proteins for the MAPP based differential ratio values of the TCPA dataset.

467 Top 10 proteins in the combined list of CS and CT based analysis were listed. In addition to

MAPP, PCPP and NCPP based values were also shown.

\begin{tabular}{|c|c|c|c|c|c|}
\hline TCPA protein symbol & $\begin{array}{l}\text { NCBI } \\
\text { symbol }\end{array}$ & $\begin{array}{l}\text { Differential } \\
\text { ratio for } \\
\text { PCPP }\end{array}$ & $\begin{array}{l}\text { Differential } \\
\text { ratio for } \\
\text { NCPP }\end{array}$ & $\begin{array}{l}\text { Differential } \\
\text { ratio for } \\
\text { MAPP }\end{array}$ & $\begin{array}{l}\text { Type of } \\
\text { analysis } \\
\text { (CS or } \\
\text { CT) }\end{array}$ \\
\hline EPPK1 & EPPK1 & -0.0149 & -0.01257 & 0.065482 & $\mathrm{CT}$ \\
\hline MYH11 & MYH11 & -0.00722 & -0.00584 & 0.05841 & $\mathrm{CT}$ \\
\hline ACETYLATUBULINLYS40 & TUBA1B & -0.00888 & -0.00596 & 0.042741 & $\mathrm{CT}$ \\
\hline ANNEXIN1 & ANXA1 & -0.00421 & -0.00371 & 0.038592 & $\mathrm{CT}$ \\
\hline TFRC & TFRC & -0.00993 & -0.00689 & 0.035851 & $\mathrm{CT}$ \\
\hline ACETYLATUBULINLYS40 & TUBA1B & -0.00732 & -0.0114 & 0.03476 & $\mathrm{CS}$ \\
\hline ANNEXIN1 & ANXA1 & -0.00894 & 0.001294 & 0.03458 & $\mathrm{CS}$ \\
\hline PEA15_pS116 & PEA15 & 0.000745 & -0.00105 & 0.032279 & $\mathrm{CT}$ \\
\hline ERALPHA & ESR1 & -0.00337 & 0.001688 & 0.029912 & CT \\
\hline PEA15_pS116 & PEA15 & -0.00695 & -0.00707 & 0.026293 & $\mathrm{CS}$ \\
\hline CAVEOLIN1 & CAV1 & -0.00609 & -0.00618 & 0.024879 & CT \\
\hline GAPDH & GAPDH & -0.01095 & -0.00856 & 0.0231 & CT \\
\hline CAVEOLIN1 & CAV1 & -0.02496 & -0.01968 & 0.017898 & $\mathrm{CS}$ \\
\hline ERALPHA & ESR1 & -0.02972 & -0.02444 & 0.015211 & $\mathrm{CS}$ \\
\hline
\end{tabular}




\begin{tabular}{|l|l|l|l|l|l|}
\hline CYCLINB1 & CCNB1 & -0.00586 & -0.00269 & 0.014624 & CT \\
\hline
\end{tabular}

Table 2. Top 10 ranked proteins for the MAPP based differential ratio values of the HPA

471 dataset. In addition to MAPP, PCPP and NCPP based values were also shown.

\begin{tabular}{|l|l|l|l|}
\hline NCBI & Differential & Differential & Differential \\
& ratio for & ratio for & ratio for \\
\hline LRRC26 & -0.00067 & -0.00033 & 0.048584 \\
\hline PC & -0.00096 & -0.00098 & 0.04155 \\
\hline ABHD3 & -0.00051 & -0.00022 & 0.035578 \\
\hline PKP3 & -0.0007 & -0.00024 & 0.035426 \\
\hline SPIDR & -0.00029 & $9.33 E-05$ & 0.02915 \\
\hline KRT5 & -0.00051 & -0.0001 & 0.028999 \\
\hline KRT17 & -0.00051 & -0.00025 & 0.028999 \\
\hline TRPS1 & -0.0003 & -0.00018 & 0.025861 \\
\hline RNASEH2C & -0.00024 & -0.00026 & 0.019282 \\
\hline GIGYF1 & -0.00054 & -0.00038 & 0.018979 \\
\hline
\end{tabular}

